\title{
A novel angiogenic pathway mediated by non-neuronal nicotinic acetylcholine receptors
}

\author{
Christopher Heeschen, ${ }^{1,2}$ Michael Weis, ${ }^{1}$ Alexandra Aicher, ${ }^{2}$ Stefanie Dimmeler, ${ }^{2}$ \\ and John P. Cooke ${ }^{1}$ \\ ${ }^{1}$ Division of Cardiovascular Medicine, Stanford University School of Medicine, Stanford, California, USA \\ ${ }^{2}$ Molecular Cardiology, Department of Internal Medicine IV, University Hospital Frankfurt, Frankfurt, Germany
}

\begin{abstract}
We have recently reported that nicotine has angiogenic effects, which appear to be mediated through non-neuronal nicotinic acetylcholine receptors (nAChRs). Here, we describe the endogenous cholinergic pathway for angiogenesis. In an in vitro angiogenesis model, increasing concentrations of the nonselective nAChR antagonist mecamylamine completely and reversibly inhibited endothelial network formation. Although several nAChR isoforms are expressed on endothelial cells (ECs), a similar inhibition was only obtained with the selective $\alpha 7$-nAChR antagonist $\alpha$-bungarotoxin, whereas other selective antagonists did not result in significant inhibition of network formation. $\alpha 7-n A C h R$ was upregulated during proliferation, by hypoxia in vitro, and by ischemia in vivo. The nAChR-induced network formation was partially dependent on VEGF, was completely dependent on the phosphatidylinositol 3-kinase and mitogen-activated protein kinase pathways, and finally resulted in NF- $\mathrm{KB}$ activation. In vivo, pharmacological inhibition of $\mathrm{nAChR}$ as well as genetic disruption of $\alpha 7-\mathrm{nACh}$ expression significantly inhibited inflammatory angiogenesis and reduced ischemia-induced angiogenesis and tumor growth. Our results suggest that $\mathrm{nAChRs}$ may play an important role in physiological and pathological angiogenesis. To our knowledge, this is the first description of a cholinergic angiogenic pathway, and it suggests a novel avenue for therapeutic modulation of angiogenesis.
\end{abstract}

J. Clin. Invest. 110:527-536 (2002). doi:10.1172/JCI200214676.

\section{Introduction}

Angiogenesis is a complex, combinatorial process that is regulated by a balance between pro- and antiangiogenic molecules (1). Angiogenic stimuli (e.g., hypoxia or inflammatory cytokines) induce the expression and release of angiogenic growth factors such as VEGF and FGF. These growth factors stimulate endothelial cells (ECs) in the existing vasculature to proliferate and to migrate through the tissue to form new endothelialized channels $(1,2)$.

We have recently demonstrated that nicotine is a potent stimulus of angiogenesis (3). This unexpected effect of nicotine appears to be mediated by nicotinic

Received for publication November 20, 2001, and accepted in revised form June 19, 2002.

Address correspondence to: John P. Cooke, Division of Cardiovascular Medicine, Stanford University School of Medicine, 300 Pasteur Drive, Stanford, California 94305, USA. Phone: (650) 725-3778; Fax: (650) 725-1599;

E-mail: john.cooke@stanford.edu.

Conflict of interest: Stanford University owns a patent on the use of nicotine receptor agonists for therapeutic angiogenesis, which has been licensed to Endovasc Inc. Authors C. Heeschen, M. Weis, and J.P. Cooke are inventors of this patent and will receive royalties from the license.

Nonstandard abbreviations used: endothelial cell (EC); nicotinic acetylcholine receptor (nAChR); human umbilical vein endothelial cell (HUVEC); human microvascular endothelial cell (HMVEC); kinase domain receptor (KDR); 3-(2,4)-

dimethoxybenzylidene anabaseine (DMXB); mitogen-activated protein kinase (MAPK); phosphatidylinositol 3-kinase (PI3K); MAP kinase kinase (MEK); pyrrolidine dithiocarbamate (PDTC); confidence interval (CI). acetylcholine receptors (nAChRs) (3). These nAChRs are ionotropic receptors, in this case agonist-regulated $\mathrm{Ca}^{2+}$ channels. Neuronal and some non-neuronal cells (e.g., bronchial epithelial cells, ECs, smooth muscle cells, and skin keratinocytes) express nAChRs (4-6). Previously, we demonstrated that nicotine stimulates angiogenesis in the settings of inflammation, ischemia, tumor, or atherosclerosis. Nicotine promoted the growth of atherosclerotic plaques and tumors at least in part by stimulating pathological angiogenesis. However, acetylcholine is the endogenous agonist of nAChRs and is synthesized and stored in ECs and blood cells, suggesting that acetylcholine may act as an autocrine factor in the cardiovascular system $(7,8)$. It is likely that endogenous acetylcholine released from ECs activates endothelial nAChRs. We embarked upon the current study to determine whether activation of nAChRs is involved in endogenous angiogenic response.

\section{Methods}

Expression of $n A C h R$. Human umbilical vein ECs (HUVECs; up to second passage) and human microvascular ECs (HMVECs; up to fourth passage; BioWhittaker Inc., Walkersville, Maryland, USA) were grown in EGM-2 supplemented with 10\% FBS (BioWhittaker Inc.). The surface expression of the nAChRs was studied in subconfluent HUVECs (EBM supplemented with 0.5\% FBS; BioWhittaker Inc.) after 12-hour nicotine stimulation $(0.1 \mathrm{nM}-1.0 \mu \mathrm{M}$; Sigma-Aldrich, St. Louis, Missouri, USA) or after exposure to hypoxia (3\% oxygen). 
We used the following antibodies $\left(1: 500\right.$ at $4^{\circ} \mathrm{C}$ for 2 hours): mouse anti- $\alpha 2$-nAChR mAb's (Sigma-Aldrich), anti- $\alpha 3-n A C h R$ mAb's (Santa Cruz Biotechnology Inc., Santa Cruz, California, USA), anti- $\alpha 4-n A C h R$ mAb's (Sigma-Aldrich), anti- $\alpha 5$-nAChR mAb's (SigmaAldrich), anti- $\alpha 7$-nAChR mAb's (Sigma-Aldrich), anti- $\beta 2$-nAChR mAb's (Sigma-Aldrich), anti- $\beta 3$-nAChR mAb's (Santa Cruz Biotechnology Inc.), and anti$\beta 4-n A C h R$ mAb's (Santa Cruz Biotechnology Inc.). After incubation with the secondary FITC-labeled goat antimouse $\mathrm{F}\left(\mathrm{ab}^{\prime}\right)_{2}$ antibody (DAKO Corp., Hamburg, Germany; $1: 4,000$ for 1 hour), cells were fixed in $1 \%$ formaldehyde/PBS and analyzed with the FACSCalibur (BD Biosciences, Franklin Lakes, New Jersey, USA). Data were analyzed using CellQuest software (Becton Dickinson), and all staining was referred to isotypematched control antibodies purchased from $\mathrm{BD}$ Pharmingen (San Diego, California, USA). Endothelial VEGF release was measured in cell culture supernatant using an ELISA for human VEGF according to the manufacturer's instructions (R\&D Systems Inc., Minneapolis, Minnesota, USA).

Tyrosine phosphorylation of VEGF receptor-2 (kinase domain receptor/Flk-1). HUVECs were starved in EBM medium containing no FCS for 12 hours. Cells were stimulated with nicotine $(0.01 \mu \mathrm{M}$ and $0.1 \mu \mathrm{M})$ or $\operatorname{VEGF}(100 \mathrm{ng} / \mathrm{ml})$ for 5 and 15 minutes. Then cells were incubated in cell lysis buffer $(20 \mathrm{mmol} / 1$ Tris [pH 7.4], $150 \mathrm{mmol} / \mathrm{l} \mathrm{NaCl}, 1 \mathrm{mmol} / 1 \mathrm{EDTA}, 1 \mathrm{mmol} / \mathrm{l}$ EGTA, $1 \%$ Triton, $2.5 \mathrm{mmol} / 1$ sodium pyrophosphate, 1 $\mathrm{mmol} / \mathrm{l} \beta$-glycerophosphate, $1 \mathrm{mmol} / 1 \mathrm{Na}_{3} \mathrm{VO}_{4}, 1 \mu \mathrm{g} / \mathrm{ml}$ leupeptin, and $1 \mathrm{mmol} / \mathrm{l}$ phenylmethylsulfonyl fluoride) for 5 minutes on ice. Cells were scraped off the plates and sonified with a Branson sonifier (Branson Ultrasonics, Danbury, Connecticut) on ice. After centrifugation for 10 minutes at $20,000 \mathrm{~g}$ at $4^{\circ} \mathrm{C}$, protein concentration in the supernatant was determined using the Bio-Rad protein assay dye reagent concentrate (BioRad Laboratories, Hercules, California, USA). Proteins $(50 \mu \mathrm{g} /$ lane) were loaded onto $8 \%$ SDS-polyacrylamide gels and blotted onto polyvinylidene fluoride membranes. After blocking with 5\% BSA at room temperature for 2 hours, the membrane was incubated with an antibody against phospho-VEGF receptor (Tyr996) (New England Biolabs Inc., Frankfurt, Germany), 1:500, at $4^{\circ} \mathrm{C}$ overnight in TBS $(50 \mathrm{mmol} / \mathrm{l}$ Tris- $\mathrm{HCl}[\mathrm{pH} 8]$, $150 \mathrm{mmol} / 1 \mathrm{NaCl}$, and $2.5 \mathrm{mmol} / \mathrm{l} \mathrm{KCl}), 0.1 \%$ Tween-20, and $5 \%$ BSA. After incubation with the second antibody (anti-rabbit, 1:4,000) for 1 hour, enhanced chemiluminescence was performed according to the instructions of the manufacturer (Amersham Biosciences, Uppsala, Sweden). The blots were reprobed with $\beta$-tubulin overnight (diluted 1:2,000 in TBS/0.1\% Tween-20/3\% BSA; Lab Vision Corp., Fremont, California, USA).

In vitro angiogenesis assay. For migration studies, 24-well plates were coated with growth factor-enhanced Matrigel (BD Biosciences, Bedford, Massachusetts, USA) and equilibrated with basal medium (EBM; BioWhittaker Inc.) (9). Cells were seeded on top of the gel at a density of $1 \times 10^{5}$ cells per well in fresh medium containing 5\% FBS. After 2 hours, increasing concentrations of nAChR antagonists $(1.0 \mathrm{pM}-1.0 \mu \mathrm{M})$ were added: mecamylamine (nonselective $\mathrm{nAChR}$ antagonist; Sigma-Aldrich), $\alpha$-bungarotoxin $(\alpha 7$-nAChR antagonist; Sigma-Aldrich), $\alpha$-conotoxin MII ( $\alpha 3 / \beta 2$-and $\beta 3$-nAChR antagonist; Tocris Cookson Inc., Ellisville, Missouri, USA), $\alpha$-lobeline $(\alpha 4 / \beta 2-n A C h R$ antagonist; Tocris Cookson Inc.), $d$-tubocurarine $(\alpha 3 / \beta 4-$ and $\alpha 4 / \beta 4-n A C h R$ antagonist; Sigma-Aldrich), and dihydro- $\beta$-erythroidine $(\alpha 3 / \beta 2$ - and $\alpha 4 / \beta 2$-nAChR antagonist; Sigma-Aldrich). Atropine (1.0 pM-1.0 $\mu \mathrm{M}$; SigmaAldrich) was used for inhibition of muscarinic acetylcholine receptors.

In other experiments, ECs seeded on growth factor-reduced Matrigel (BD Biosciences, Bedford, Massachusetts, USA) were treated with the nAChR agonist nicotine $(0.1 \mu \mathrm{M}$; Sigma-Aldrich) or the $\alpha 7$-selective nicotinic receptor agonist 3-(2,4)-dimethoxybenzylidene anabaseine (DMXB; $1.0 \mu \mathrm{M})$, respectively. To investigate whether VEGF and/or basic FGF (bFGF) are involved in nAChR-mediated angiogenesis, we used neutralizing anti-VEGF $(0.1-5 \mu \mathrm{g} / \mathrm{ml}$; SigmaAldrich) and anti-bFGF mAb's $(0.1-5 \mu \mathrm{g} / \mathrm{ml}$; Upstate Biotechnology Inc., Lake Placid, New York, USA), respectively. Endothelial VEGF release was measured in cell culture supernatant using an ELISA for human VEGF according to the manufacturer's instructions (R\&D Systems Inc.). Downstream mediators of nAChR stimulation were investigated using the p38 mitogen-activated protein kinase (MAPK) inhibitor SB203580 (Alexis Biochemicals, Grünberg, Germany), the MAP kinase kinase (MEK) $1 / 2$ inhibitor PD98059 (Biomol Research Laboratories, Hamburg, Germany), the phosphatidylinositol 3-kinase (PI3K) inhibitor LY294002 (Calbiochem-Novabiochem, San Diego, California, USA), and the NF- $\mathrm{kB}$ inhibitor pyrrolidine dithiocarbamate (PDTC) (Sigma-Aldrich). Protein tyrosine kinase inhibition was obtained using herbimycin A (1 $\mu \mathrm{M}$; Sigma-Aldrich).

Development of tube formation was investigated at 24,48 , and 72 hours using an inverted phase-contrast microscope (Zeiss AxioVert 100M; Carl Zeiss Inc., Oberkochen, Germany). Images were captured with a video graphic system (Zeiss AxioCam; Carl Zeiss Inc.). Relative tube formation was determined by measuring the length of tubes in five random fields from each well and is expressed as percent of vehicle-treated cells.

Disc angiogenesis system. A disc of polyvinyl alcohol sponge (Rippey Corp., El Dorado Hills, California, USA), covered with nitrocellulose cell-impermeable filters (Millipore Corp., Burlington, Massachusetts, USA), allows capillaries to grow only through the rim of the $\operatorname{disc}(3,10,11)$. The randomized treatment with either mecamylamine $(5 \mu \mathrm{g} / \mathrm{kg})$ or PBS for the 3-week study period was delivered via osmotic minipumps $(0.25 \mu \mathrm{l} / \mathrm{h}$; Durect Corp., Cupertino, California). The discs were subcutaneously implanted in the backs of 10 -week-old C57BL/6J mice ( $n=8$ per group) or $\alpha 7-\mathrm{nAChR}^{-/-}$mice 
(B6.129S7-Chrna7tm1Bay; $n=6$ per group; The Jackson Laboratory, Bar Harbor, Maine, USA). Three weeks later, mice were anesthetized and space-filling fluorescent microspheres $(0.2 \mu \mathrm{m}$; Molecular Probes Inc., Eugene, Oregon, USA) were injected into the left ventricle to deliver them to the systemic microvasculature (12). Both the area of the disc invested by fibrovascular growth and the vessel density were assessed $(3,11)$. The expression of $\alpha 7$-nAChRs in vessels growing into the disc was identified by polyclonal antibodies against mouse $\alpha 7$-nAChR (Santa Cruz Biotechnology Inc.; 1:500 at $4^{\circ} \mathrm{C}$ overnight) followed by preadsorbed phycoerythrin-labeled goat anti-rabbit antibodies (Molecular Probes Inc.; $1: 1,000$ for 2 hours at $4^{\circ} \mathrm{C}$ ). To verify the specificity of the staining, discs explanted from $\alpha 7-\mathrm{nAChR}^{-/-}$mice were used as a negative control.

Murine model of hind limb ischemia. In 10-week-old C57BL/6J mice, the distal end of the external iliac artery and the deep and superficial femoral artery were ligated ( $n=8$ animals per group) (13). Mecamylamine was administered for 3 weeks either locally by intramuscular injections into the ischemic hind limb on a daily basis $(0.05$ and $0.5 \mu \mathrm{g} / \mathrm{kg})$ or systemically by osmotic minipumps subcutaneously implanted in the backs of the mice $(5.0 \mathrm{mg} / \mathrm{kg}$ delivered over a 3 -week period).

Capillary density was determined in the adductor and semimembranous muscles. In $10-\mu \mathrm{m}$ cryostat cross sections through the midbelly of the muscle, capillaries were identified by mAb's against CD31 (BD PharMingen), and myocytes were counterstained with eosin (Sigma-Aldrich). Capillary density for each section was determined in ten randomly selected fields and is expressed as a ratio of capillaries to myocytes. The $\alpha 7$-nAChRs in those sections were identified by immunohistochemistry using the same staining protocol as described above (anti- $\alpha 7-n A C h R$ antibodies from Santa Cruz Biotechnology Inc.). Again, sections from $\alpha 7-\mathrm{nAChR}^{-/-}$mice served as a negative control.

For Western blotting, muscle tissue from ischemic and nonischemic limbs ( $n=4$ for each time point) was homogenized in ice-cold sample buffer and centrifuged at $10,000 \mathrm{~g}$ for 15 minutes at $4^{\circ} \mathrm{C}$, and the supernatant was recentrifuged at $200,000 \mathrm{~g}$ for 30 minutes at $4^{\circ} \mathrm{C}$. Pellets were then resuspended in icecold sample buffer, and protein concentrations were determined by the method of Lowry et al. with 1 $\mathrm{mg} / \mathrm{ml} \mathrm{BSA}$ as a standard (14). The protein extracts were dissolved 1:1 (vol/vol) in Laemmli buffer and, 50 $\mu \mathrm{g}$ of protein was loaded on each lane of $8 \%$ SDSPAGE. The proteins were then transferred by electroblotting onto PVDF membranes. The membranes were incubated overnight at $4^{\circ} \mathrm{C}$ with $0.5 \mu \mathrm{g} / \mathrm{ml}$ (1:400) of primary antibodies for $\alpha 7-\mathrm{nAChR}$ (Santa Cruz Biotechnology Inc.) and then incubated with horseradish peroxidase-labeled, preadsorbed antigoat secondary antibodies (1:6,000; Pierce Chemical Co., Rockford, Illinois, USA) for 2 hours at room temperature. The blots were exposed to Hyperfilm ECL (Amersham), and the integrated optical density of each band and gel background was measured using Scion Image (Scion Corp., Frederick, Maryland, USA). Antibodies against $\alpha 7-\mathrm{nAChR}$ produced one major band at $57 \mathrm{kDa}(15)$.

Lewislung tumor model. A total of $10^{6}$ Lewis lung carcinoma cells (American Type Culture Collection, Manassas, Virginia, USA) were cultured in RPMI 1640 supplemented with $10 \%$ FBS. A total of $10^{6}$ cancer cells were subcutaneously injected unilaterally into the right flank or orthotopically introduced into the right lung parenchyma via a limited skin incision without thoracotomy of 10 -week-old C57BL/6J wildtype mice. Animals received mecamylamine $(3 \mathrm{mg} / \mathrm{kg})$ or vehicle via osmotic minipumps implanted on the left flank of the mice. Mice were checked on a daily basis for the presence of a palpable tumor, and tumor growth was measured with a skin caliper. Tumor size was calculated as the product of length $/ 2 \times$ width $/ 2 \times$ thickness $/ 2 \times(4 / 3) \pi \mathrm{cm}^{3}$. When mice in any group were bearing tumors greater than $1.5 \mathrm{~cm}^{3}$, the experiment was stopped and all animals sacrificed for histological evaluation. Tumor vessels were identified by infusion of space-filling fluorescent microspheres $(0.2$ $\mu \mathrm{m}$; Molecular Probes Inc.) via the left ventricle of the heart as described above. Serum VEGF levels were determined with a mouse VEGF ELISA kit (R\&D Systems Inc.). The surface expression of the nAChR was studied in proliferating Lewis lung cancer cells (RPMI 1640 with $10 \%$ FBS) with and without 12-hour nicotine stimulation $(0.1 \mu \mathrm{M}$ and $1.0 \mu \mathrm{M}$; Sigma-Aldrich).

Statistical analysis. All results for continuous variables are expressed as medians with $95 \%$ confidence intervals (CIs). In the figures, the medians are presented along with $75 \%$ and $95 \%$ CI. Comparisons between groups were analyzed by $t$ test (two-sided) or ANOVA for experiments with more than two subgroups. Post hoc range tests and pairwise multiple comparisons were performed with the $t$ test (two-sided) with Bonferroni adjustment. Comparison of categorical variables was generated by the Pearson $\chi^{2}$ test. All analyses were performed with SPSS 10.0 (SPSS Inc., Chicago, Illinois, USA). $P$ values less than 0.05 were considered statistically significant.

\section{Results}

Expression of nicotinic acetylcholine receptors on ECs. We first performed tests to determine which nAChRs are expressed on ECs. Consistent with previous studies, several isoforms are expressed on proliferating (subconfluent) HUVECs (Figure 1a) and HMVECs (data not shown). Staining for the nAChR subunits $\alpha 3, \alpha 4, \alpha 7$, $\beta 2$, and $\beta 4$ resulted in a significant signal in the FACS analysis, whereas the nAChR subunits $\alpha 2, \alpha 5$, and $\beta 3$ remained undetectable (Figure 1a). One of the most abundant nAChRs expressed in proliferating HUVECs was the $\alpha 7$-nAChR (Figure 1b). In confluent ECs, however, this receptor was significantly downregulated (-62\% [95\% CI 43-84\%]) (Figure 1b). Interestingly, treatment with nicotine further increased the expression of 


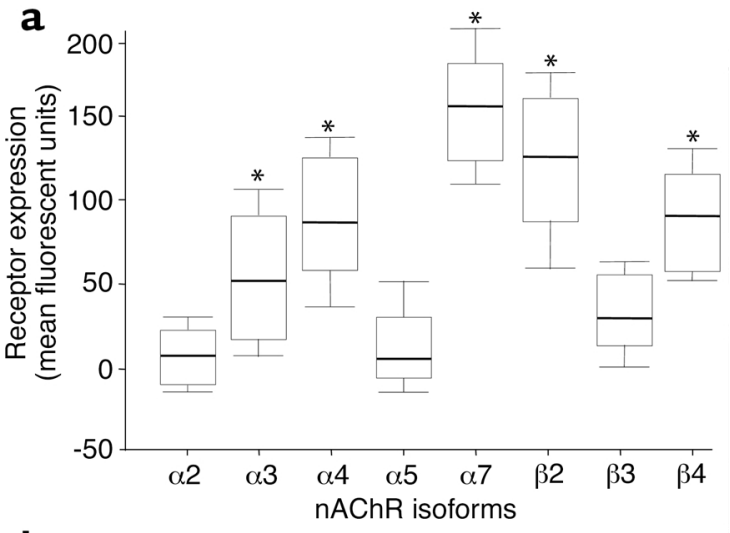

b

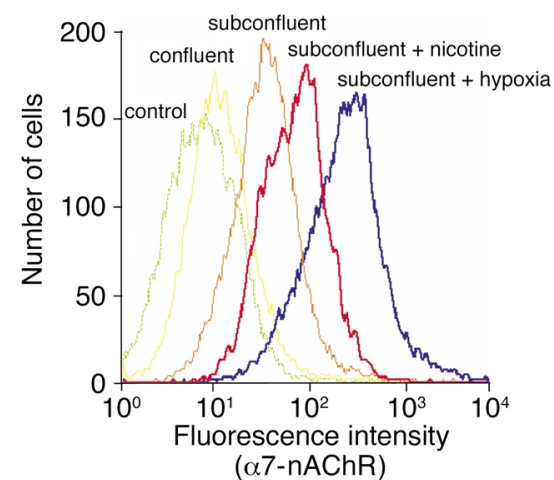

\section{Figure 1}

Expression of $\mathrm{nAChR}$ subunits on subconfluent ECs. (a) FACS analysis demonstrated that the subunits $\alpha 3, \alpha 4, \alpha 7, \beta 2$, and $\beta 4$ were expressed on the utilized ECs. Results are shown as box plots displaying 25th and 75th percentiles as boxes and 5th and 95th percentiles as thin lines. $n=4$ for each $\mathrm{nAChR}$ subunit; ${ }^{*} P<0.01$ compared with isotype control. (b) The $\alpha 7-n A C h R$ was detectable at very low levels on confluent ECs. The expression of $\alpha 7-n A C h R$ was significantly increased during proliferation (in subconfluent cells), during hypoxia (4 hours), or during stimulation with nicotine $(0.1 \mathrm{nM}$, 12 hours). A representative histogram is shown. GAM/PE, secondary goat anti-mouse antibody labelled with phycoerythrin.

the $\alpha 7-\mathrm{nAChR}(+57 \%$ [38-94\%]), as did the exposure to hypoxia (+161\% [88-235\%]) (Figure 1b). The nAChR isoforms $\alpha 4$ and $\beta 2$ showed a similar pattern, whereas expression of other isoforms did not significantly change under the above conditions (data not shown). Effect of $n A C h R$ on tube formation. After seeding the cells on growth factor-enriched Matrigel, ECs manifested tube formation within 48 hours in response to control medium (Figure 2, a and g). However, when the medium was supplemented with increasing concentrations of mecamylamine $(10 \mathrm{pM}-10 \mu \mathrm{M})$, a specific noncompetitive inhibitor of $\mathrm{nAChR}$, network formation was significantly reduced (Figure 2, b-d). A 50\% reduction in tube formation was observed at a mecamylamine concentration of $0.1 \mu \mathrm{M}$. If mecamylamine treatment $(1.0 \mu \mathrm{M})$ was stopped after 36 hours, ECs were still capable of arranging in network-like structures at 72 hours, indicating that the effect of mecamylamine was reversible (Figure 2e). Interestingly, we were able to mimic this effect of general nAChR inhibition on network formation with selective inhibition of the $\alpha 7$-nAChR by $\alpha$-bungarotoxin. A 50\% inhibition was obtained with $1 \mathrm{nM} \alpha$-bungarotoxin. A concentration of $10 \mathrm{nM}$ inhibited capillary network formation to a degree similar to that caused by mecamylamine ( $P=0.78$ vs. mecamylamine) (Figure 2, $\mathrm{f}$ and $\mathrm{g}$ ). In contrast, other selective $\mathrm{nAChR}$ antagonists such as the $\alpha 3 / \beta 2$ - and $\beta 3$-nAChR antagonist $\alpha$-conotoxin MII, the $\alpha 4 / \beta 2$-nAChR antagonist $\alpha$-lobeline, the $\alpha 3 / \beta 4$ - and $\alpha 4 / \beta 4-\mathrm{nAChR}$ antagonist d-tubocurarine, and the $\alpha 3 / \beta 2$ - and $\alpha 4 / \beta 2$-nAChR antagonist dihydro$\beta$-erythroidine showed no significant inhibition of capillary network formation (Figure 2f). Atropine, a nonselective inhibitor of muscarinic acetylcholine receptors, also did not inhibit network formation (105\% [95\% CI $95.2-116.8 \%], P=1.00)$. These data indicate an important role of the $\alpha 7$-nAChR for angiogenesis. In line with these data, a selective $\alpha 7$-nAChR agonist, DMXB, reproducibly stimulated network formation as compared with control (Figure 3a).

To investigate whether stimulation of nAChR might influence angiogenesis via interfering with VEGF synthesis, we determined VEGF levels in the cell culture supernatant by ELISA. Treatment of the cells with nicotine $(0.1 \mu \mathrm{M})$ or the selective $\alpha 7$-nAChR agonist DMXB $(1.0 \mu \mathrm{M})$ for 48 hours induced a small but significant increase in VEGF levels (control, 228 [95\% CI 162-295] $\mathrm{pg} / \mathrm{ml}$; nicotine, 336 [251-398] pg/ml; DMXB, 298 $[198-354] \mathrm{pg} / \mathrm{ml} ; P=0.036)$. Furthermore, a minor but significant reduction of nicotine- or DMXB-stimulated network formation was observed by co-incubation with neutralizing anti-VEGF antibodies (Figure 3a). However, capillary network formation was still greater as compared with that in control-treated cells, indicating VEGF-independent effects of nAChR stimulation on network formation $(P=0.021)$. To investigate whether the partial inhibitory effect of neutralizing VEGF antibodies might be explained by the stimulation of other growth factors such as bFGF, cells were additionally incubated with neutralizing bFGF antibodies. Interestingly, the addition of bFGF-neutralizing antibodies did not result in a significant reduction of nAChR-induced tube formation (Figure 3a). Moreover, supplementation of both neutralizing antibodies did not show an additive effect (Figure 3a). Consistent with a more specific interference with VEGF signaling, the nAChR antagonist mecamylamine significantly inhibited VEGF-stimulated network formation but did not affect bFGF-induced tube formation (Figure 3b).

Since blockade of VEGF resulted in only partial inhibition of the effect of nAChR stimulation and mecamylamine also significantly inhibited network formation in the presence of excess of VEGF, we further investigated a potential effect of the $\mathrm{nAChR}$ on activation of the VEGF receptor- 2 kinase domain receptor/Flk-1 (KDR/Flk-1) by detecting KDR tyrosine phosphorylation (16). Nicotine stimulated KDR tyrosine phosphorylation at Tyr996 to an extent similar to that brought about by VEGF (Figure 3c). The tyrosine phosphorylation of KDR by nicotine was maximal 
already after 15 minutes of incubation (Figure 3c), indicating a cross-talk between the nAChR and the VEGF receptor-2. Consistently, the stimulatory effect of nicotine on network formation was partially inhibited by pretreatment of ECs with the tyrosine kinase inhibitor herbimycin $\mathrm{A}$, which was shown to block VEGF effects in ECs (control, 100\% [95\% CI 88-117\%]; nicotine, $232 \%$ [181-274\%]; nicotine + herbimycin A, $170 \%$ [134-205\%]; $P<0.01)(17)$.

We further examined the signaling pathways mediating $\mathrm{nAChR}$-induced tube formation. To investigate the involvement of MAPK extracellular signal-regulated kinase and p38 MAPK, we used PD98059, an MAPK inhibitor, and SB203580, an inhibitor of p38 MAPK. Both PD98059 and SB203580, but not the vehicle, inhibited the nAChR-induced increase in network formation (Figure 3d). Likewise, nAChR-mediated stimulation of network formation was completely dependent on activation of PI3K/Akt pathways, as shown by abrogation of network formation by the PI3K inhibitor LY294002. In addition, tube formation was also sensitive to PDTC, an inhibitor of NF- $\mathrm{KB}$, suggesting that downstream signaling of $\mathrm{nAChR}$ activation finally involves in NF- $\mathrm{KB}$ activation.

Disc angiogenesis system. To determine whether $\mathrm{nAChRs}$ are involved in the native angiogenic response in vivo, we used the disc angiogenesis system. In the control group, 54.9\% [95\% CI 46.1-63.6\%] of the cross-sectional area of the disc was covered with new vessels when the disc was removed 3 weeks after subcutaneous implantation (Figure 4, a, b, and e). Systemic treatment with mecamylamine decreased neovascularization of the disc by $75 \%$ (to $14.3 \%$ of the cross-sectional area of the disc [95\% CI 9.0-19.5\%]; $P<0.001$ ) (Figure 4, c, d, and e). In contrast, atropine, a nonselective inhibitor of muscarinic acetylcholine receptors, did not inhibit neovascularization of the disk (Figure 4e). Immunohistochemistry for $\alpha 7$-nAChR indicated expression of this receptor in proliferating vessels in the disc (Figure 4f).

In homozygous $\alpha 7-\mathrm{nAChR}^{-1-}$ mice, the native angiogenic response was reduced from 39.5\% (95\% CI 28.7-44.8\%) for the $\alpha 7-\mathrm{nAChR}^{+/+}$wild-type control mice to $28.7 \%$ (95\% CI $25.2-32.2 \%$; $P=0.009$ ) (Figure $4 \mathrm{~g}$ ). However, nicotine increased fibrovascular growth in the knockout as well as the wild-type mice. Nicotine administration via daily subcutaneous injections $(10 \mu \mathrm{g} / \mathrm{d})$ increased fibrovascular growth in the $\alpha 7-\mathrm{nAChR}^{+/+}$ wild-type control mice (to $55.3 \%$ of the cross-sectional area of the disc [95\% CI 49.4-63.3\%]; $P=0.001)$ and to a lesser extent in the homozygous $\alpha 7-\mathrm{nAChR}^{-/-}$mice (to $34.2 \%$ of the cross-sectional area of the disc [95\% CI 31.1-43.2\%]; $P=0.021$ ).

Murine model of hind limb ischemia. To determine whether nAChRs play an important role in the setting of angiogenesis in response to ischemia, we employed the murine model of hind limb ischemia (13). After unilateral ligation of the superficial and deep femoral arteries, we administered mecamylamine $(0.05 \mu \mathrm{g} / \mathrm{kg}$ or $0.5 \mu \mathrm{g} / \mathrm{kg})$, atropine $(0.05 \mu \mathrm{g} / \mathrm{kg})$, or vehicle by intramuscular injections into the ischemic hind limb on a daily basis for a period of 3 weeks $(n=8$ per group). After 3 weeks of treatment, $0.5 \mu \mathrm{g} / \mathrm{kg}$ mecamylamine significantly reduced the angiogenic response from 0.45 capillaries per myocyte $(95 \% \mathrm{CI}$ $0.39-0.51)$ to 0.26 capillaries per myocyte $(95 \% \mathrm{CI}$ $0.21-0.31 ; P<0.001)$ (Figure 5a). We achieved similar results when we administered mecamylamine systemically via osmotic minipumps (5.0 $\mu \mathrm{g} / \mathrm{kg}$ over 14 days) that were subcutaneously implanted in the back $(0.24$ capillaries per myocyte [95\% CI 0.19-0.28]; $P<0.001$ ). We did not observe a change in capillary density in the nonischemic hind limb when we administered
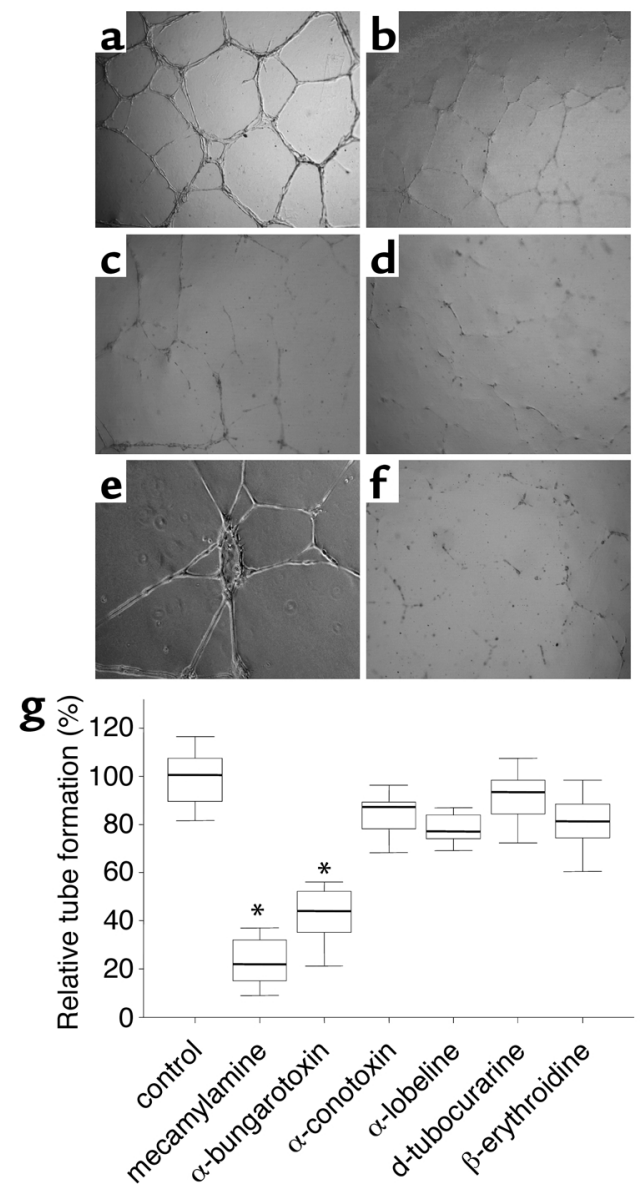

Figure 2

Effect of nAChR inhibition on angiogenesis in vitro. (a) Seeding of ECs on Matrigel resulted in capillary network formation within 48 hours. (b-d) Mecamylamine dose-dependently inhibited network formation: $1 \mathrm{nM}(\mathbf{b}), 100 \mathrm{nM}(\mathbf{c})$, and $1 \mu \mathrm{M}(\mathbf{d})$. (e) If mecamylamine $(1 \mu \mathrm{M})$ was removed after 36 hours, the effect of mecamylamine on in vitro angiogenesis was reversible and endothelial networks had formed by 72 hours. Representative microscopic pictures are shown. (f) This effect of mecamylamine on network formation was mimicked by the $\alpha 7-n A C h R$ inhibitor $\alpha$-bungarotoxin ( $1 \mathrm{nM}$ ). (g) Other selective $\mathrm{nAChR}$ antagonists did not result in a significant reduction of network formation. Results are shown as box plots displaying 25 th and 75 th percentiles as boxes and 5 th and 95 th percentiles as thin lines $\left(n=4 ;{ }^{*} P<0.001\right.$ vs. control. Data are expressed as a percentage of network formation under control conditions, i.e., in the presence of vehicle alone. 

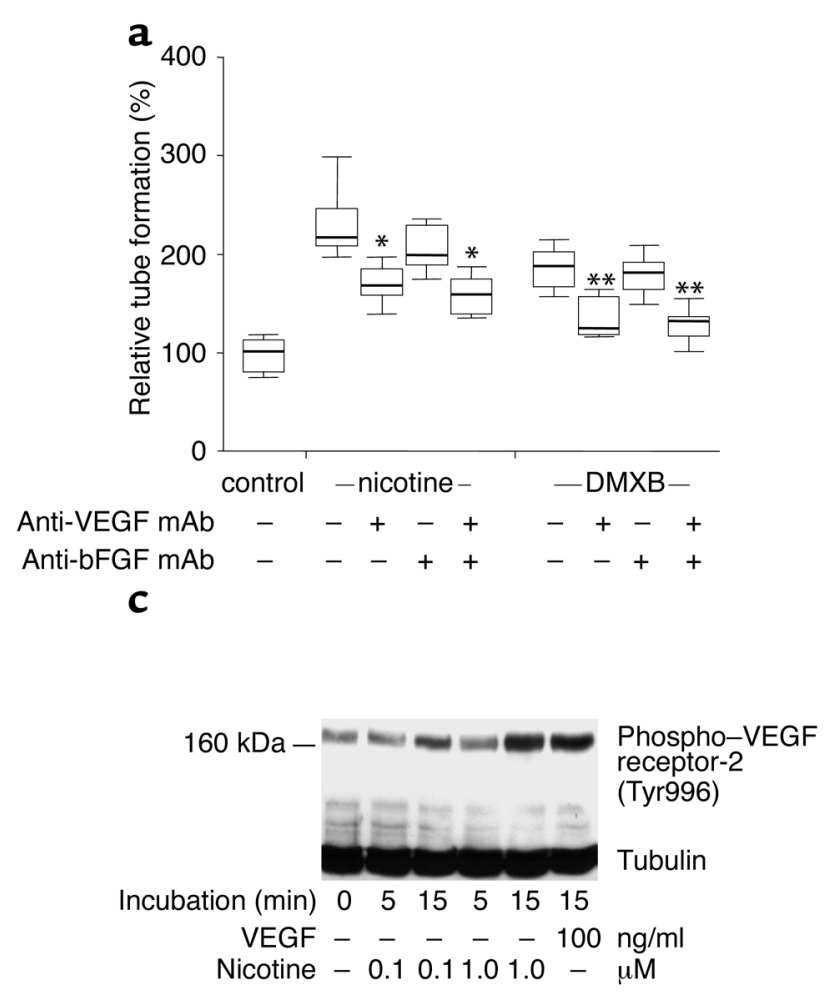

b
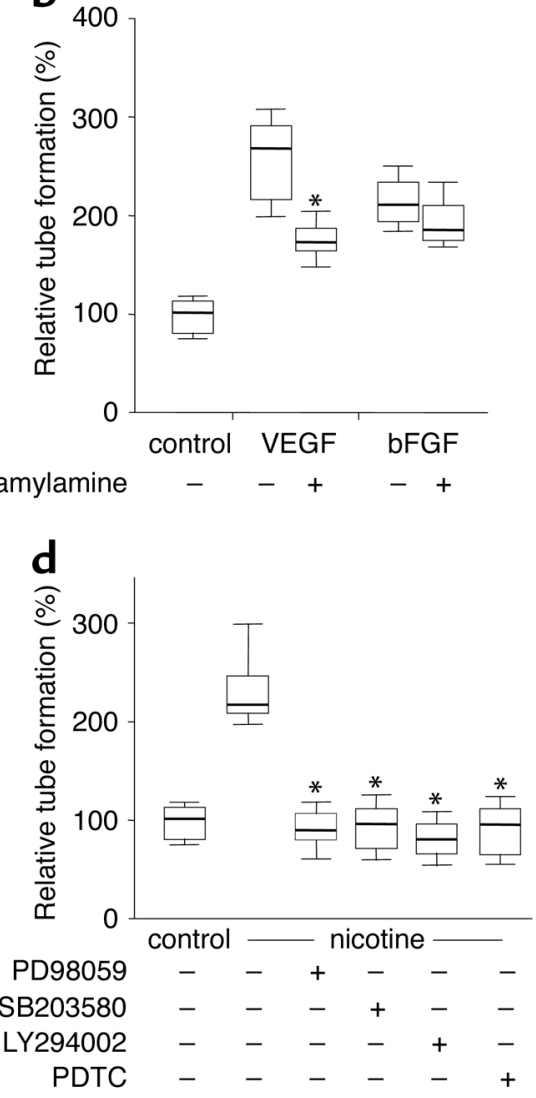

Figure 3

Mechanism of nAChR-mediated angiogenesis. (a) VEGF-neutralizing antibodies, but not bFGF-neutralizing antibodies, partially inhibit nAChRinduced network formation. ${ }^{*} P<0.01$ vs. nicotine alone; ${ }^{*} P<0.01$ vs. DMXB alone. (b) The nAChRs are partially involved in VEGF-mediated angiogenesis but not in bFGF-mediated angiogenesis. ${ }^{*} P<0.01 \mathrm{vs}$. VEGF alone. (c) Nicotine dose- and time-dependently stimulates VEGF receptor-2 activation. A representative blot is shown. $n=3$. (d) The MAPK (MEK) inhibitor PD98059, the p38 MAPK inhibitor SB203580, the PI3K inhibitor LY294002, and the NF-KB inhibitor PDTC completely inhibited nAChR-induced network formation. Results are shown as box plots displaying 25th and 75th percentiles as boxes and 5th and 95th percentiles as thin lines. ${ }^{*} P<0.01$ vs. nicotine alone. $n=4$ for each experiment.

mecamylamine systemically $(P=0.97)$, locally into the ischemic hind limb $(P=0.88)$, or directly into the nonischemic hind limb $(P=0.51)$. Atropine, administered either locally or systemically, did not alter neovascularization of the ischemic hind limb (Figure 5a).

An increase in capillary density does not necessarily enhance local blood flow if not associated with a concomitant increase in collateral vessels. Accordingly, we identified collaterals by double staining for CD31 (ECs) and $\alpha$-actin (smooth muscle cells) using confocal microscopy. The number of collaterals was quantified on cross sections by identifying vascular lumen lined by CD31-positive endothelial cells that were coated by an actin-positive vascular smooth muscle wall and had vessel diameters of at least $0.02 \mathrm{~mm}$. Compared with the nonischemic hind limb, the number of collateral vessels in the ischemic limb of the control animals significantly increased from 2.6 (95\% CI $2.5-4.3)$ to $7.5(95 \%$ CI 5.2-9.5; $P<0.001)$ and the median size of the collateral vessels increased from $0.05 \mathrm{~mm}(95 \%$ CI $0.02-0.11)$ to $0.12 \mathrm{~mm}$ (95\% CI $0.08-0.15 ; P<0.001)$. Treatment with mecamylamine did not significantly decrease the number of collateral vessels (high dose, 6.2 [95\% CI 3.9-8.6]; low dose, 7.1 [95\% CI 4.7-8.5]; $P=0.08$ ). However, the median diameter of the vessels significantly decreased (high dose to $0.07 \mathrm{~mm}$ [95\% CI 0.04-0.10]; low dose to $0.09 \mathrm{~mm}$ [95\% CI 0.06-0.11]; $P=0.002$ ).

Immunohistochemistry for the $\alpha 7$-nAChRs demonstrated that this receptor subunit is not expressed at detectable levels in nonischemic hind limbs (Figure 5, b and c). In contrast, 7 days after induction of ischemia, this receptor subtype clearly colocalized with some of the ECs, as identified by CD31 (Figure 5, $\mathrm{d}$ and e). We were able to confirm this upregulation of the $\alpha 7$ subunit in ischemic tissue by Western blot analysis (Figure $5 f)$. The $\alpha 7$ subunit was undetectable at base line, but only 6 hours after induction of hind limb ischemia this receptor subtype was significantly upregulated with a maximum at 7 days.

Murine model of lung cancer. Previous studies indicate that nAChRs play an important role in angiogenesis in a broad spectrum of experimental conditions (3). To determine whether mecamylamine could influence 
tumor angiogenesis, we used the in vivo Lewis lung cancer model. Up to 12 days after implantation of the cancer cells and treatment with vehicle or mecamylamine, all mice exhibited similar tumor size $\left(0.46 \mathrm{~cm}^{2}\right.$ [95\% CI $0.32-0.56$ ] vs. $0.49 \mathrm{~cm}^{2}$ [95\% CI $\left.0.35-0.62\right] ; P=0.54$ ) (Figure 6). At 21-day follow-up, however, tumor growth in the control group significantly exceeded that observed in the mecamylamine group and required sacrifice of the animals $\left(1.21 \mathrm{~cm}^{3}\right.$ [95\% CI $\left.0.82-1.36\right]$ vs. $0.85 \mathrm{~cm}^{3}$ [95\% CI 0.66-1.05]; $\left.P<0.001\right)$. This late decrease in tumor growth in the mecamylamine group corresponded with a reduced vascularization of the tumor tissue. We observed a significantly lower capillary density for the mecamylamine group (0.82 capillaries $/ \mathrm{mm}^{2}$ [95\% CI $0.67-1.22$ ] vs. 0.56 capillaries $/ \mathrm{mm}^{2}$ [95\% CI 0.38-0.92]; $P<0.001$ ). In another experiment, we orthotopically implanted Lewis lung cancer cells into the lung parenchyma. Larger tumors in the control group required discontinuation of the experiment after 17 days (tumor volume of $0.62 \mathrm{~cm}^{3}$ [95\% CI $0.37-0.85$ ] vs. $0.45 \mathrm{~cm}^{3}$ [95\% CI 0.28-0.66]; $\left.P<0.001\right)$. Again, tumor vascularization was significantly lower in the mecamylamine group (1.56 capillaries $/ \mathrm{mm}^{2}$ [95\% CI 0.82-2.17] vs. 0.98 capillaries $/ \mathrm{mm}^{2}$ [95\% CI 068-1.31]; $\left.P<0.001\right)$. Furthermore, the systemic levels of VEGF were significantly higher in the control group than in the mecamylamine group (152.2 pg/ml [95\% CI 98.5-187.2] vs. 78.7 $\mathrm{pg} / \mathrm{ml}$ [95\% CI 45.3-99.4]; $P<0.001)$.

FACS analysis revealed that the immortalized Lewis lung cancer cell line does not express any nAChR subunits (data not shown). In subsequent cell culture experiments, we investigated whether the observed reduction in tumor growth could still be related to a direct antiproliferative effect of mecamylamine on the cancer cells. After up to 48 hours of treatment with mecamylamine, we did not observe a significant change in the number of Lewis lung cancer cells $(10.2$ [95\% CI 8.2-13.4] $\times 10^{5}$ cells as compared with $10.6[95 \% \mathrm{CI}$ 8.4-13.8] $\times 10^{5}$ cells for control; $\left.n=4 ; P=0.84\right)$.

\section{Discussion}

The salient findings of our work are: (a) Inhibition of nAChRs significantly and reversibly reduces capillary network formation of cultured ECs; (b) nAChR-mediated neovascularization is mainly mediated by $\alpha 7-n A C h R$; (c) inhibition of $\mathrm{nAChRs}$ reduces the angiogenic response to inflammation (disc angiogenesis model), ischemia (femoral artery ligation model), and neoplasia (Lewis lung cancer model); (d) the angiogenic effect of $\mathrm{nAChR}$ activation involves MAPK, PI3K/Akt, and NF- $\kappa \mathrm{B}$ activation; and, intriguingly, (e) the nAChR and VEGF receptor appear to mediate distinct but interdependent pathways of angiogenesis. These data reveal, for the first time to our knowledge, that nAChRs are involved in the native angiogenic response, and that this pathway is distinct from those triggered by VEGF or FGF.

Recent patch clamp studies demonstrated that human ECs express functional nAChRs (5). Endothelial nAChRs are ligand-gated ionotropic channels consisting of $\alpha$ subunits or a combination of $\alpha$ and $\beta$ subunits, each of which has a unique pharmacological profile (18). We found that the nAChR isoforms $\alpha 3$,
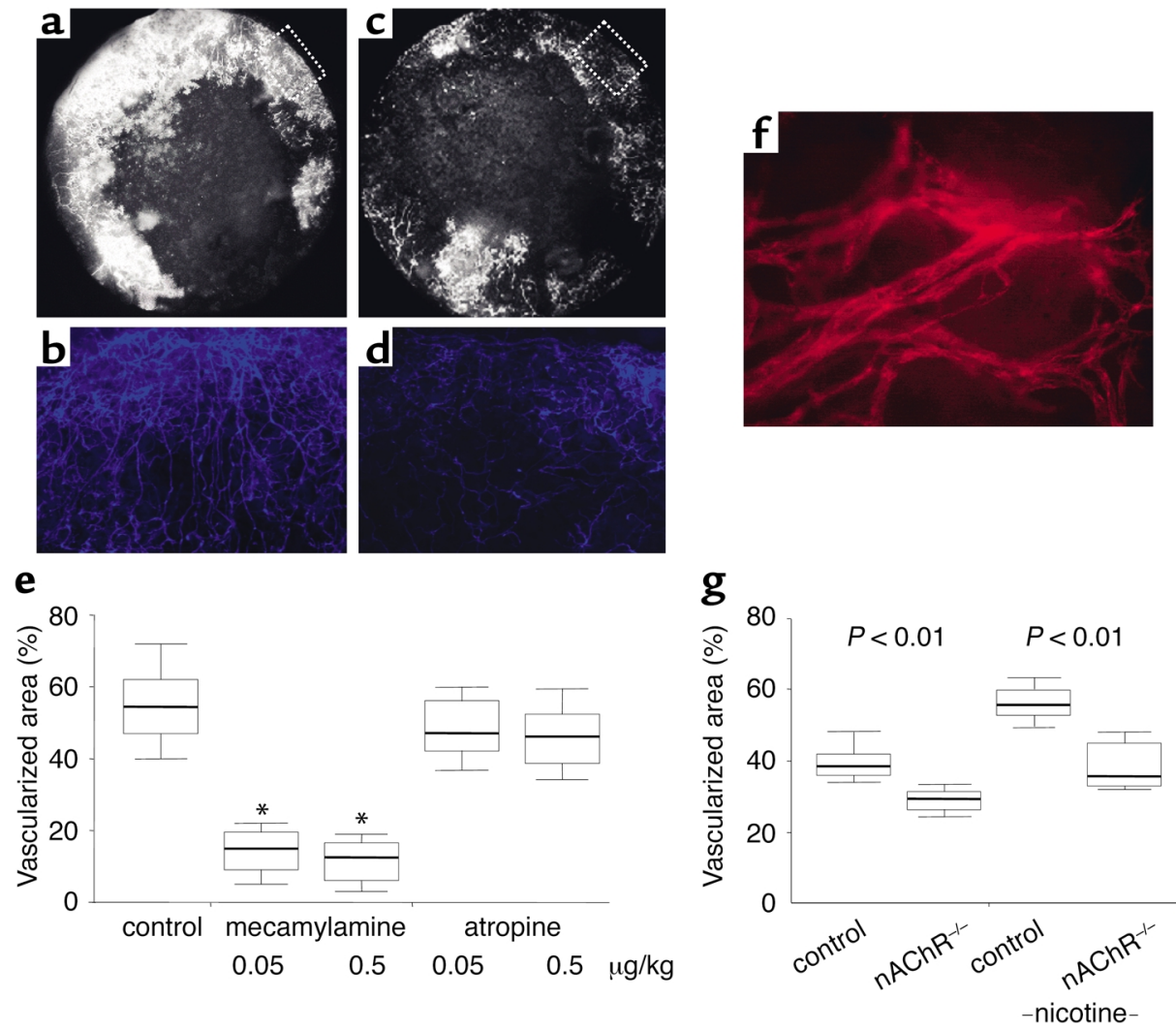

\section{Figure 4}

Disc angiogenesis system: mecamylamine inhibits inflammatory angiogenesis in vivo. (a and $\mathbf{b}$ ) Subcutaneous implantation of a polyvinyl sponge for 3 weeks resulted in ingrowth of new vessels ( $b$ is a detailed view of the tagged area in a). (c-e) The nAChR antagonist mecamylamine significantly decreased the vascularized area of the disc (c and $\mathbf{e}$ ) and the vessel density in the disc ( $\mathbf{d}$, a detailed view of the tagged area in $\mathbf{c}$ ). In contrast, treatment with the muscarinic acetylcholine receptor antagonist did not result in a significant reduction of neovascularization (e). ${ }^{*} P<0.01$ vs. control. (f) Proliferating vessels in the disc stained positive for the $\alpha 7-n A C h R$. A representative microscopic picture is shown. (g) In $\alpha 7$-nAChR-deficient mice, the angiogenic response was significantly reduced but the proangiogenic effect of nicotine was partially preserved. Results are shown as box plots displaying 25 th and 75 th percentiles as boxes and 5 th and 95 th percentiles as thin lines. $n=5 ; P<0.01$ compared with control mice. 

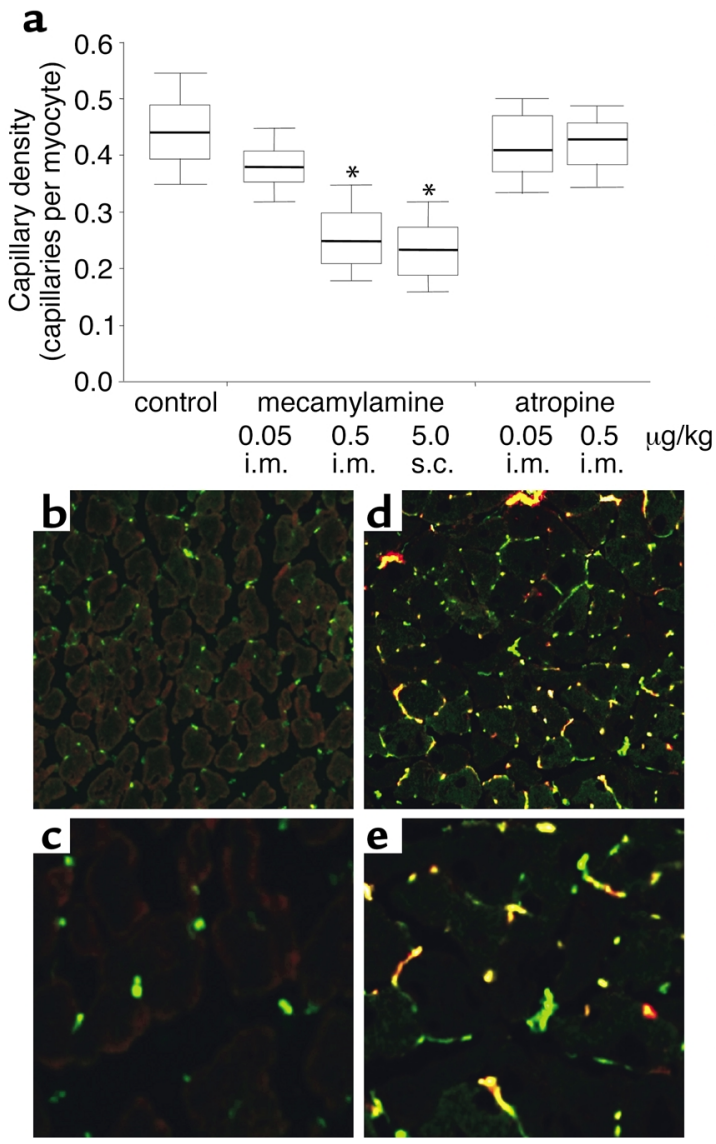

f

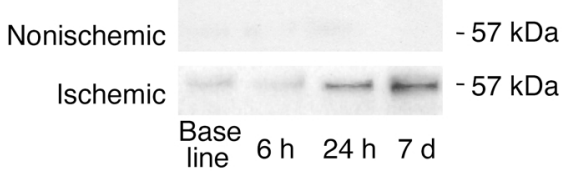

$\alpha 4, \alpha 7, \beta 2$, and $\beta 4$ were each expressed by HUVECs and HMVECs. The most abundant receptor subunit was the $\alpha 7$-nAChR, which recently has been found to be expressed by ECs (19). Homomeric $\alpha 7$-nAChRs are strikingly different from their heteromeric counterparts. Expression of $\alpha 7$-encoding RNA generates acetylcholine-evoked currents sensitive to nanomolar concentrations of $\alpha$-bungarotoxin, a toxin that is without effect on $\alpha / \beta$-type nAChR. The $\alpha 7$-nAChR is also characterized by exceptionally high permeability to $\mathrm{Ca}^{2+}$ and rapid desensitization in response to high concentrations of nicotinic agonists (20). However, recent studies reveal that $\alpha 7$-nAChRs

\section{Figure 6}

Lewis lung tumor model: mecamylamine inhibits advanced tumor growth. Two weeks after subcutaneous injection of Lewis lung cancer cells, tumor growth was similar in the control and mecamylamine groups. In the subsequent study period, however, animals treated with mecamylamine showed significantly less tumor growth. Data are median $(95 \% \mathrm{Cl}) ; n=5 ; P<0.01$ compared with control at 24 days. Tumor vascularity was suppressed by mecamylamine (data not shown).

\section{Figure 5}

Murine model of hind limb ischemia: mecamylamine inhibits ischemia-induced angiogenesis. Saline or increasing concentrations of mecamylamine were administered by daily intramuscular injections into the ischemic hind limb. (a) As compared with control, mecamylamine decreased capillary density. Results are shown as box plots displaying 25th and 75th percentiles as boxes and 5th and 95th percentiles as thin lines. $n=5$; ${ }^{*} P<0.01$ vs. control. i.m., intramuscular; s.c., subcutaneous. (b and c) At base line (no ischemia), the $\alpha 7$ subunit (red fluorescence) was not detectable by immunohistochemistry (capillaries identified by CD31, green fluorescence). (d and e) However, 7 days after induction of ischemia in the hind limb and no treatment, the $\alpha 7$ subunit of the nAChR was clearly detectable (red fluorescence) and mainly colocalized with CD31 (yellow fluorescence). Representative pictures are shown. (f) These results were confirmed by Western blot analyses demonstrating upregulation of the $\alpha 7-n A C h R$ subunit within 24 hours after onset of ischemia and a maximal effect at 7 days.

desensitize very slowly in response to sustained application of a low concentration of acetylcholine, nicotine, or other nAChR agonists (21). Hence, the kinetics of agonist delivery and the local concentration of agonist are important determinants of the overall effect of $\alpha 7$-nAChR stimulation. Interestingly, we observed a marked upregulation of $\alpha 7$-nAChRs in hypoxic ECs as early as 4 hours after induction of hypoxia. We also found an upregulation of the $\alpha 7-n A C h R s$ in capillaries of the ischemic hind limb and in the setting of inflammatory angiogenesis (disc angiogenesis model). In contrast, we could not detect the presence of $\alpha 7$-nAChRs in the vasculature of nonischemic limbs. In vitro studies with HUVECs demonstrated that this receptor is expressed at very low levels in confluent ECs. In subconfluent cultures ( $50 \%$ confluence) we observed a significant increase in the $\alpha 7-\mathrm{nAChR}$ expression. Consistent with the in vivo findings, we observed a marked upregulation of the $\alpha 7-\mathrm{nAChRs}$ in HUVECs after treatment with nicotine and in particular after exposure to hypoxia (Figure 1b). Thus, the $\alpha 7$-nAChRs are upregulated in conditions associated with angiogenesis.

Previous work by Villablanca and colleagues provided in vitro evidence that nicotine, an exogenous agonist of the nAChR, stimulates EC proliferation (22). We have recently shown that stimulation of the

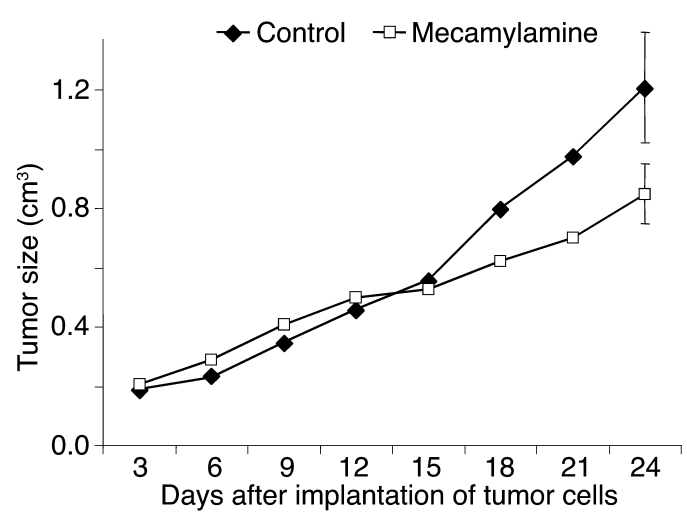


nAChR with nicotine reduces EC apoptosis and causes morphological changes in the endothelial monolayer consistent with an angiogenic agent (3). We found that nicotine induces angiogenesis in a number of milieus, including inflammation, ischemia, tumor, and atherosclerosis. The angiogenic effects of nicotine were mediated by $\mathrm{nAChR}$, as the specific nAChR antagonists mecamylamine and hexamethonium, respectively, abolished the proangiogenic effect of nicotine. Recent evidence suggests that non-neuronal nAChRs are involved in the regulation of vital cell functions, such as mitosis, differentiation, organization of the cytoskeleton, cell-cell contact, locomotion, and migration (6). Thus, acetylcholine may function as a local "hormone" that is able to modulate cell functions that require adaptation to new conditions (18). Our findings suggest that angiogenic response to ischemia may involve activation of the nicotinic signaling pathway. The $\alpha 7-\mathrm{AChR}$ appears to play a crucial role in this pathway. The selective $\alpha 7-n A C h R$ antagonist $\alpha$-bungarotoxin inhibited capillary network formation in vitro, as did the nonselective nAChR antagonist mecamylamine (Figure $2 b$ ). Treatment with other selective nAChR antagonists did not significantly inhibit capillary network formation in vitro. The proangiogenic effect of nicotine was mimicked by the selective $\alpha 7-\mathrm{nAChR}$ agonist DMXB. We further investigated the role of the $\alpha 7-\mathrm{nAChR}$ in vivo using $\alpha 7-\mathrm{nAChR}^{-/-}$mice. The knockout mice exhibited an attenuated angiogenic response to ischemia and inflammation (Figure 4g). However, the angiogenic response associated with genetic abrogation of the $\alpha 7$-nAChR (-27\%) was less than that observed with pharmacological inhibition of the $\alpha 7-\mathrm{nAChR}(-75 \%)$. This may be due to developmental upregulation or adaptation of other nAChRs in the knockouts. It is known that nicotine also binds to $\beta 2$-nAChR $(\alpha 3 / \beta 2$ and $\alpha 4 / \beta 2-n A C h R)$. Indeed, we observed that pharmacological inhibition of $\mathrm{nAChR}$ containing $\beta 2$ subunits tended to slightly reduce network formation in vitro, an effect that did not reach statistical significance (Figure $2 \mathrm{~g}$ ). This alternative pathway could explain why the knockout mice could respond (albeit in a diminished manner) to nicotine with an increase in angiogenic response.

The nAChR-mediated stimulation of angiogenesis was completely dependent on activation of PI3K/Akt pathways, as shown by abrogation of network formation by the PI3K inhibitor LY294002. In addition, tube formation was also sensitive to PDTC, an inhibitor of $\mathrm{NF}-\mathrm{KB}$, suggesting that downstream signaling of $\mathrm{nAChR}$ activation finally results in NF- $\mathrm{KB}$ activation. Interestingly, Kihara et al. were able to demonstrate that the neuroprotective effect of nicotine was also dependent on PI3K activity and that nicotine increased Akt phosphorylation, an effector of PI3K. Consistent with our results, Kihara et al. observed that the neuroprotective effect was inhibited by $\alpha$-bungarotoxin and was mimicked by the $\alpha 7$-nAChR agonist DMXB, indicating a crucial role for the $\alpha 7$-nAChRs in neuronal cells (23). Phosphorylation of eNOS via the Akt pathway represents an important $\mathrm{Ca}^{2+}$-dependent regulatory mechanism for activation of eNOS $(24,25)$. The present study indicates that $\mathrm{nAChR}$ stimulation may also regulate Akt in the cardiovascular system and, thus, activate eNOS and other downstream targets of Akt (26-28). These findings are also consistent with recent in vivo studies demonstrating that nAChR stimulation increases production of nitric oxide $(29,30)$.

To determine whether nAChR-mediated angiogenesis was mediated by VEGF or bFGF we used neutralizing antibodies. Neutralization of VEGF resulted in a significant but not complete inhibition of nAChR-mediated network formation. Neutralizing antibodies against bFGF had no significant effect on nAChR-mediated network formation. Further studies demonstrated that nAChR stimulation and selective stimulation of $\alpha 7-\mathrm{nAChR}$ both resulted in a significant release of VEGF. In addition, nAChR stimulation resulted in transactivation of VEGF receptor-2, as demonstrated by Western blot analysis (Figure 3c). Notably, antagonists of the nAChR markedly attenuated VEGF-induced angiogenesis. These results are most consistent with two distinct but interdependent pathways for angiogenesis.

In conclusion, we provide in vitro and in vivo evidence that nicotinic acetylcholine receptors mediate angiogenesis in response to diverse stimuli. These studies indicate for the first time, to our knowledge, that there is an endogenous cholinergic pathway for angiogenesis. This cholinergic pathway may play a role in pathological as well as therapeutic angiogenesis. Modulation of the activity of this pathway may represent a new therapeutic avenue for disorders characterized by inadequate or pathological angiogenesis.

\section{Acknowledgments}

We thank Christiane Mildner-Rihm for excellent technical assistance. This study was supported in part by grants from the National Heart, Lung, and Blood Institute (R01 HL-58638), the Tobacco-Related Disease Research Program (7RT-0128), Phillip Morris Inc., and the German Research Council (He 3044/1-1). J.P. Cooke is an Established Investigator of the American Heart Association.

\footnotetext{
1. Carmeliet, P., and Jain, R.K. 2000. Angiogenesis in cancer and other diseases. Nature. 407:249-257.

2. Folkman, J. 1995. Angiogenesis in cancer, vascular, rheumatoid and other disease. Nat. Med. 1:27-31.

3. Heeschen, C., et al. 2001. Nicotine stimulates angiogenesis and promotes tumor growth and atherosclerosis. Nat. Med. 7:833-837.

4. Grando, S.A., et al. 1995. A nicotinic acetylcholine receptor regulating cell adhesion and motility is expressed in human keratinocytes. J. Invest. Dermatol. 105:774-781.

5. Macklin, K.D., Maus, A.D., Pereira, E.F., Albuquerque, E.X., and ContiFine, B.M. 1998. Human vascular endothelial cells express functional nicotinic acetylcholine receptors. J. Pharmacol. Exp. Ther. 287:435-439. 6. Wessler, I., Kirkpatrick, C.J., and Racke, K. 1999. The cholinergic "pitfall": acetylcholine, a universal cell molecule in biological systems, including humans. Clin. Exp. Pharmacol. Physiol. 26:198-205.

7. Kawashima, K., Oohata, H., Fujimoto, K., and Suzuki, T. 1989. Extraneuronal localization of acetylcholine and its release upon nicotinic stimulation in rabbits. Neurosci. Lett. 104:336-339.
} 
8. Kawashima, K., et al. 1990. Synthesis and release of acetylcholine by cultured bovine arterial endothelial cells. Neurosci. Lett. 119:156-158.

9. Kureishi, Y., et al. 2000. The HMG-CoA reductase inhibitor simvastatin activates the protein kinase Akt and promotes angiogenesis in normocholesterolemic animals. Nat. Med. 6:1004-1010.

10. Fajardo, L.F., Kowalski, J., Kwan, H.H., Prionas, S.D., and Allison, A.C. 1988. The disc angiogenesis system. Lab. Invest. 58:718-724.

11. Jang, J.J., Ho, H.K., Kwan, H.H., Fajardo, L.F., and Cooke, J.P. 2000 Angiogenesis is impaired by hypercholesterolemia: role of asymmetric dimethylarginine. Circulation. 102:1414-1419.

12. Springer, M.L., Ip, T.K., and Blau, H.M. 2000. Angiogenesis monitored by perfusion with a space-filling microbead suspension. Mol. Ther 1:82-87.

13. Couffinhal, T., et al. 1998. Mouse model of angiogenesis. Am. J. Pathol. 152:1667-1679.

14. Lowry, O.H., Rosebgrough, N.J., Farr, A.L., and Randall, R.J. 1959. Protein measurement with the Folin phenol reagent. J. Biol. Chem. 153:265-275.

15. Martin-Ruiz, C.M., et al. 1999. Alpha4 but not alpha3 and alpha7 nicotinic acetylcholine receptor subunits are lost from the temporal cortex in Alzheimer's disease. J. Neurochem. 73:1635-1640.

16. Meyer, M., et al. 1999. A novel vascular endothelial growth factor encoded by Orf virus, VEGF-E, mediates angiogenesis via signalling through VEGFR-2 (KDR) but not VEGFR-1 (Flt-1) receptor tyrosine kinases. EMBO J. 18:363-374.

17. Murohara, T., et al. 1998. Vascular endothelial growth factor/vascular permeability factor enhances vascular permeability via nitric oxide and prostacyclin. Circulation. 97:99-107.

18. Conti-Fine, B.M., Navaneetham, D., Lei, S., and Maus, A.D. 2000. Neuronal nicotinic receptors in non-neuronal cells: new mediators of tobacco toxicity? Eur. J. Pharmacol. 393:279-294.
19. Wang, Y., et al. 2001. Human bronchial epithelial and endothelial cells express alpha7 nicotinic acetylcholine receptors. Mol. Pharmacol. 60:1201-1209.

20. McGehee, D.S., and Role, L.W. 1995. Physiological diversity of nicotinic acetylcholine receptors expressed by vertebrate neurons. Annu. Rev. Physiol. 57:521-546.

21. Fenster, C.P., Rains, M.F., Noerager, B., Quick, M.W., and Lester, R.A. 1997. Influence of subunit composition on desensitization of neuronal acetylcholine receptors at low concentrations of nicotine. J. Neurosi. 17:5747-5759.

22. Villablanca, A.C. 1998. Nicotine stimulates DNA synthesis and proliferation in vascular endothelial cells in vitro. J. Appl. Physiol. 84:2089-2098.

23. Kihara, T., et al. 2001. Alpha 7 nicotinic receptor transduces signals to phosphatidylinositol 3-kinase to block A beta-amyloid-induced neurotoxicity. J. Biol. Chem. 276:13541-13546.

24. Fulton, D., et al. 1999. Regulation of endothelium-derived nitric oxide production by the protein kinase Akt. Nature. 399:597-601.

25. Dimmeler, S., et al. 1999. Activation of nitric oxide synthase in endothelial cells by Akt-dependent phosphorylation. Nature. 399:601-605.

26. Dimmeler, S., and Zeiher, A.M. 2000. Akt takes center stage in angiogenesis signaling. Circ. Res. 86:4-5.

27. Franke, T.F., Kaplan, D.R., and Cantley, L.C. 1997. PI3K: downstream AKTion blocks apoptosis. Cell. 88:435-437.

28. Matsui, T., et al. 2001. Akt activation preserves cardiac function and prevents injury after transient cardiac ischemia in vivo. Circulation. 104:330-335

29. Clouse, W.D., et al. 2000. Effects of transdermal nicotine treatment on structure and function of coronary artery bypass grafts. J. Appl. Physiol. 89:1213-1223.

30. Green, J.T., et al. 2000. Nitric oxide mediates a therapeutic effect of nicotine in ulcerative colitis. Aliment. Pharmacol. Ther. 14:1429-1434. 